\title{
Substantia Nigra Free Water Increases Longitudinally in Parkinson Disease
}

\author{
(D)T. Guttuso Jr, (D) N. Bergsland, (D). Hagemeier, DD.G. Lichter, (D) Pasternak, and (D) R. Zivadinov
}

\begin{abstract}
BACKGROUND AND PURPOSE: Free water in the posterior substantia nigra obtained from a bi-tensor diffusion MR imaging model has been shown to significantly increase over 1- and 4-year periods in patients with early-stage idiopathic Parkinson disease compared with healthy controls, which suggests that posterior substantia nigra free water may be an idiopathic Parkinson disease progression biomarker. Due to the known temporal posterior-to-anterior substantia nigra degeneration in idiopathic Parkinson disease, we assessed longitudinal changes in free water in both the posterior and anterior substantia nigra in patients with later-stage idiopathic Parkinson disease and age-matched healthy controls for comparison.
\end{abstract}

MATERIALS AND METHODS: Nineteen subjects with idiopathic Parkinson disease and 19 age-matched healthy control subjects were assessed on the same 3T MR imaging scanner at baseline and after approximately 3 years.

RESULTS: Baseline mean idiopathic Parkinson disease duration was 7.1 years. Both anterior and posterior substantia nigra free water showed significant intergroup differences at baseline $(P<.001$ and $P=.014$, respectively, idiopathic Parkinson disease versus healthy controls); however, only anterior substantia nigra free water showed significant longitudinal group $\times$ time interaction increases $(P=.021$, idiopathic Parkinson disease versus healthy controls). There were no significant longitudinal group $\times$ time interaction differences found for conventional diffusion tensor imaging or free water-corrected DTI assessments in either the anterior or posterior substantia nigra.

CONCLUSIONS: Results from this study provide further evidence supporting substantia nigra free water as a promising disease-progression biomarker in idiopathic Parkinson disease that may help to identify disease-modifying therapies if used in future clinical trials. Our novel finding of longitudinal increases in anterior but not posterior substantia nigra free water is potentially a result of the much longer disease duration of our cohort compared with previously studied cohorts and the known posterior-to-anterior substantia nigra degeneration that occurs over time in idiopathic Parkinson disease.

ABBREVIATIONS: aSN = anterior substantia nigra; $\mathrm{FW}=$ free water; $\mathrm{HC}=$ healthy control; IPD = idiopathic Parkinson disease; IPD-NM = subjects with IPD not receiving any $\mathrm{MAOI}$ at baseline; IPD-R = subjects with IPD receiving rasagiline at baseline; $\mathrm{MAOI}=$ monoamine oxidase inhibitor; $\mathrm{MMSE}=\mathrm{Mini}-\mathrm{Mental}$ State Examination; MoCA = Montreal Cognitive Assessment; $\mathrm{MNI}=$ Montreal Neurological Institute; $\mathrm{NODDI}=$ neurite orientation dispersion and density imaging; $\mathrm{pSN}=$ posterior substantia nigra; $\mathrm{SN}=$ substantia nigra pars compacta

$A^{\prime}$ $\mathrm{t}$ the time of diagnosis of idiopathic Parkinson disease (IPD) about $30 \%-50 \%$ of the dopaminergic neurons in the sub-

Received July 3, 2017; accepted after revision November 19.

From the Movement Disorder Center (T.G., D.G.L.) and Buffalo Neuroimaging Analysis Center (N.B., J.H., R.Z.), Department of Neurology, and MR Imaging Clinical and Translational Research Center (R.Z.), Jacobs School of Medicine and Biomedical Sciences, University at Buffalo, State University of New York, Buffalo, New York; and Departments of Psychiatry and Radiology (O.P.), Brigham and Women's Hospital, Harvard Medical School, Boston, Massachusetts.

This work was supported by an investigator-initiated award from Teva Neurosciences and from the National Center for Advancing Translational Sciences of the National Institutes of Health, ULITR001412, and National Institutes of Health awards R01MH108574 and P41EB015902.

Please address correspondence to Thomas Guttuso Jr, MD, 3435 Main St, 97 Farber Hall, Buffalo, NY 14214; e-mail: tguttuso@buffalo.edu

- Indicates open access to non-subscribers at www.ajnr.org stantia nigra pars compacta $(\mathrm{SN})$ have degenerated, resulting in dopamine deficiency and motor symptoms. ${ }^{1-3}$ This degeneration is initially focused in the ventrolateral tier, which is grossly in the posterior $\mathrm{SN}$, with the more anterior regions of the $\mathrm{SN}$ affected relatively later in the disease. ${ }^{3}$ Thus, the pattern of SN neuronal loss in IPD is heterogeneous and progresses from a posterior-toanterior direction with over time.

Diffusion tensor imaging is a diffusion MR imaging-derived assessment that reflects tissue microstructural integrity. ${ }^{4}$ As a result, multiple groups have sought to determine whether SN DTI can distinguish subjects with IPD from healthy controls (HCs). Results of these studies have been conflicting. ${ }^{5}$ These conflicting results have been theorized to be due to inconsistent methodologies used to de-

http://dx.doi.org/10.3174/ajnr.A5545 
lineate the SN or unpredictable contamination of DTI assessments from free water (FW), which is extracellular water. ${ }^{5-7} \mathrm{FW}$ mapping obtained from a bi-tensor diffusion MR imaging model was developed, which allows separating out the contribution of FW to DTI assessments (FW-corrected DTI) and for determining the fraction of FW itself, to control for FW contamination. ${ }^{7}$ Most interesting, subsequent studies have shown that FW itself in the posterior (p) SN distinguishes patients with IPD from HCs better than conventional DTI as well as FW-corrected DTI assessments. ${ }^{8,9}$ Furthermore, 2 studies by the Vaillancourt group have shown pSN FW to significantly increase longitudinally over 1 and 4 years in patients with early-stage IPD compared with HCs, suggesting that pSN FW may be a disease-progression biomarker in early IPD. ${ }^{10,11}$

Due to the known temporal posterior-to-anterior SN degeneration in IPD, ${ }^{3}$ we assessed longitudinal changes in FW and explored changes in FW-corrected DTI and conventional DTI in both the posterior and anterior (a) SN in patients with later-stage IPD and age-matched HCs over approximately 3 years. Additional exploratory outcomes included assessment for associations between longitudinal changes in clinical outcomes (motor and cognitive) and longitudinal changes in DTI/FW in the SN and multiple other regions of interest. We were particularly interested in identifying ROIs that may reflect longitudinal worsening of cognition because dementia eventually occurs in $80 \%$ of patients with IPD, is likely unrelated to SN pathology, and is one of the most disabling long-term sequela of IPD. ${ }^{12}$ Finally, we also explored differences in these outcomes between the subjects with IPD receiving the monoamine oxidase inhibitor (MAOI) rasagiline at baseline and IPD subjects not receiving any $\mathrm{MAOI}$ at baseline to examine the potential disease-modifying actions of rasagiline. ${ }^{13}$

\section{MATERIALS AND METHODS}

The institutional review board at the University at Buffalo provided ethics approval for this study before subject enrollment. From March 2011 to March 2013, thirty-two subjects with IPD and 25 age- and sex-matched HC subjects provided written informed consent and underwent MR imaging and clinical assessment, including 'on' state Unified Parkinson's Disease Rating Scale Part III, ${ }^{14}$ the Montreal Cognitive Assessment (MoCA), ${ }^{15}$ and the Mini-Mental State Examination (MMSE). ${ }^{16}$ Subsequently, from January to October 2015, after again providing written informed consent, available subjects were re-assessed with the same clinical and MR imaging measures (follow-up assessments). All subjects with IPD satisfied the UK Parkinson's Disease Society Brain Bank criteria for diagnosis ${ }^{17}$ and were assessed clinically by the same movement disorder neurologist (T.G. or D.G.L.) at both time points. Healthy controls consisted primarily of spouses and friends of the participants with IPD. A small number of healthy controls in this study had participated in previous research studies at the University at Buffalo and had consented to be contacted for future studies.

Using the same 3T-MR imaging scanner Signa Excite HD 12.0 Twin Speed 8-channel; (GE Healthcare, Milwaukee, Wisconsin) and a multichannel head and neck coil at both time points, we performed diffusion MR imaging with a voxel size of $2 \times 2 \times 4$ $\mathrm{mm}^{3}$ and a $0.5-\mathrm{mm}$ gap. The sequence used 2 averages with 25 noncollinear directions, $b=900 \mathrm{~s} / \mathrm{mm}^{2}$ and 1 volume without directional weighting $\left(b=0 \mathrm{~s} / \mathrm{mm}^{2}\right)$. A high-resolution $3 \mathrm{D}$ T1- weighted volume with $1 \times 1 \times 1 \mathrm{~mm}^{3}$ isotropic voxels was also acquired. Image analyses were performed in the Buffalo Neuroimaging Analysis Center by an evaluator (N.B.) blinded to subjects' clini$\mathrm{cal}$ and treatment characteristics. DTI image analyses were performed with the FSL 5.0 Toolbox (www.fmrib.ox.ac.uk/fsl). We used code implemented in Matlab (MathWorks, Natick, Massachusetts) to fit a regularized bi-tensor model and to generate FW maps and FW-corrected maps of the 4 standard DTI assessments. ${ }^{7}$ To eliminate all non-neural tissue, we extracted the original $b=0$ images (with no diffusion-weighting) and de-skulled them in FSL using the Brain Extraction Tool (http://fsl.fmrib.ox.ac.uk/fsl/fslwiki/BET). Susceptibility-induced geometric distortions were corrected for using nonlinear registration of the $b=0 \mathrm{~s} / \mathrm{mm}^{2}$ image to the T1WI volume, while eddy currents were corrected using the FSL eddy tool (https://fsl.fmrib.ox.ac.uk/fsl/fslwiki/eddy). ${ }^{18}$

FW, FW-corrected DTI, and conventional DTI measures of fractional anisotropy, mean diffusivity, axial diffusivity, and radial diffusivity were assessed in the anterior and pSN as well as several regions likely to reflect cognitive status ${ }^{19-23}$ : the anterior and posterior cingulate, anterior thalamus, fornix, genu and splenium of the corpus callosum, and major lobar white matter tracts. All ROIs were drawn manually except for those in the lobar white matter. The former ROIs were derived by nonlinear registration of the 3D T1WI volume Montreal Neurological Institute (MNI) space. MNI-defined lobar masks were warped into native space using the corresponding inverse warp. Finally, the ROIs were then restricted to voxels having a partial volume estimate of $>0.85$ for white matter, as derived from tissue segmentation of the 3D T1WI volume. ${ }^{24}$

SN ROIs were drawn by one of the blinded authors (N.B.) as previously described. ${ }^{11}$ Briefly, the $b=0$ image was linearly registered with $12 d f$ to a T2-weighted MNI space image with $2-\mathrm{mm}^{3}$ isotropic voxels. ${ }^{25}$ Next, left and right hemisphere anterior and posterior SN ROIs were drawn separately on each $b=0$ image after transformation into MNI space. Each ROI consisted of a $2 \times 2 \mathrm{~mm}$ square placed on 2 consecutive slices, for a total of eight $2 \times 2 \times 2 \mathrm{~mm}^{3}$ voxels. The superior slice for the SN ROI placement was chosen just inferior to the red nucleus where the red nucleus was either barely or no longer visible. Anterior and pSN ROIs were placed in the area of hypointense signal on the $b=0$ image corresponding to the SN (Fig 1). Such SN ROI placement is also consistent with placement guided by neuromelanin-sensitive imaging. ${ }^{5}$ Finally, the DTI-derived maps were resampled into MNI space with trilinear interpolation using the $b=0$ transformation matrix, and quantitative measures were extracted from the SN ROIs. Other exploratory ROIs were delineated as previously described. ${ }^{19,21,23}$ The MR imaging scanner underwent no hardware or software upgrades during the study.

Statistical analyses were conducted using SPSS for Windows, Version 24.0 (IBM, Armonk, New York). Intergroup differences in baseline characteristics were analyzed using the $\chi^{2}$ test, Student $t$ test, and nonparametric Mann-Whitney $U$ test when appropriate. The repeated measures ANCOVA general linear model framework with age and sex as covariates was used for longitudinal and cross-sectional assessments. The ROI main effects of group and time as well as the interaction effect of group $\times$ time were considered significant at $P<.05$. There were no corrections for multiple comparisons in non-SN ROIs due to the exploratory nature of these outcomes and the limited sample size of the study. 


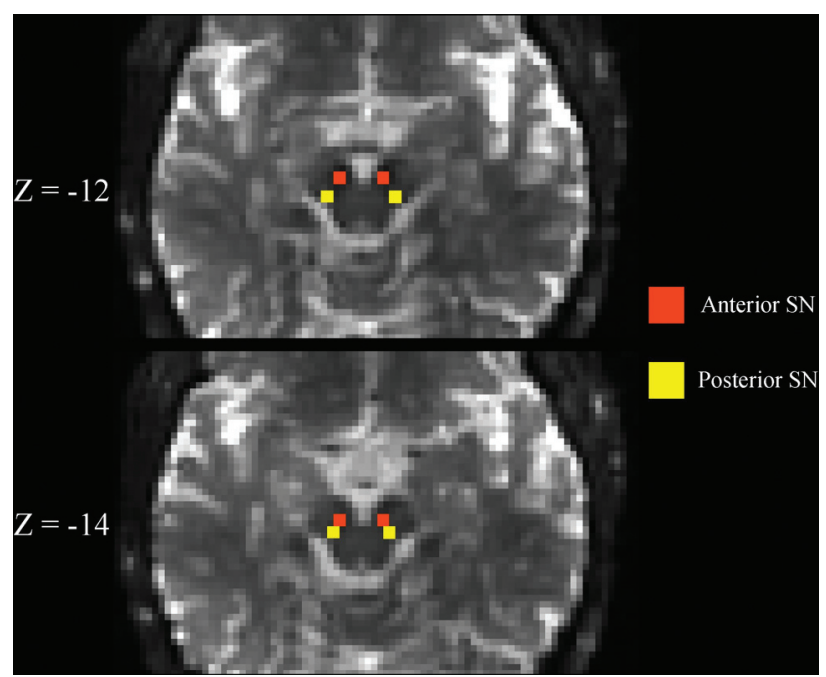

FIG 1. Substantia nigra ROls. Representative $b=0 \mathrm{~s} / \mathrm{mm}^{2}$ images linearly registered to the $\mathrm{MNI}$ atlas are shown demonstrating the placement of the substantia nigra ROls. Z-coordinates refer to the slice shown in MNI space. See the "Materials and Methods" section for a more detailed description of how ROls were placed.

Within-Parkinson-disease-group associations between longitudinal changes in imaging measures and longitudinal changes in clinical outcomes were assessed using age-, sex- and disease duration-adjusted forward-selection models $(P<.05$ selection criterion), in which change in clinical outcomes was considered the dependent measure. Post hoc analyses for differences at baseline between the subjects with IPD receiving rasagiline at baseline (IPD-R) and subjects with IPD not receiving any MAOI at baseline (IPD-NM) were performed using the Fisher least significant difference and Fisher exact test (categoric) and for longitudinal differences using the repeated measures ANCOVA general linear model framework with disease duration as a covariate. For reproducibility analysis of the manually drawn ROIs, 2-way absolute agreement, single-measure intraclass correlation coefficient was used. A random sample of data from 5 healthy controls and 5 patients with IPD was analyzed and re-analyzed after 1 month to minimize recall bias. The order of the analyses was also randomized.

\section{RESULTS}

Of the original 57 subjects enrolled at baseline, 18 were unable to be reassessed at the 3-year follow-up visit due to death $(n=4)$, a deep brain stimulation operation $(n=2)$, change in residence $(n=3)$, decision not to participate $(n=6)$, or loss to follow-up $(n=3)$. Another subject was removed from the analyses after a frontal lobe astrocytoma was found on the 3-year follow-up MR imaging. Therefore, 38 subjects with available data at both time points were included in the analyses. The characteristics of these 38 subjects are summarized in Table 1. There were no significant intergroup differences in subjects' ages, but the IPD group had significantly more men and a longer time to follow-up compared with the HC group.

Anterior SN FW demonstrated both significant intergroup differences at baseline and group $\times$ time longitudinal interaction differences over the 3-year follow-up period, while pSN FW only demonstrated significant intergroup differences at baseline (Fig 2 and Table 2). No other SN DTI or FW-corrected DTI assessment showed both significant intergroup and group $\times$ time interaction differences.
Comparisons of longitudinal changes in MR imaging assessments with longitudinal changes in clinical outcomes showed increasing bitemporal lobe white matter FW-corrected mean diffusivity to be associated with decreasing (worsening) MMSE and MoCA scores (Fig 3; standardized coefficient, $P$ values; respectively: -0.909 , $P=.001 ;-0.534, P=.037)$. In addition, left aSN FW and total pSN FW changes were associated with worsening MMSE but not MoCA scores (standardized coefficient, $P$ values; respectively: $-0.781, P=$ $.002 ; 0.563, P=.011)$. Only increasing left parietal lobe white matter FW-corrected axial diffusivity was associated with increasing (worsening) Unified Parkinson's Disease Rating Scale Part III motor scores (standardized coefficient, $P$ value: $0.654, P=.033$ ).

There were no significant baseline or longitudinal differences between the IPD-R $(n=11)$ and IPD-NM $(n=8)$ groups for any ROI assessment after correction for disease duration (5.1 years for subjects with IPD-R and 9.8 years for those with IPD-NM at baseline). The subjects with IPD-R had been receiving rasagiline for a mean of $35.6 \pm 40.3$ months before baseline.

For the left anterior $\mathrm{SN}$, left posterior $\mathrm{SN}$, right anterior $\mathrm{SN}$, and right posterior $\mathrm{SN}$, the intraclass correlation coefficient values were $0.93,0.85,0.91$, and 0.83 , respectively. Intraclass correlation coefficient results of the non-SN ROIs ranged between 0.75 (for the anterior thalamus) to 0.96 (for the genu of the corpus callosum).

\section{DISCUSSION}

In our later-stage IPD cohort, we found significant longitudinal intergroup differences in aSN FW, with more rapid increases in subjects with IPD compared with HCs over approximately 3 years of follow-up (Fig 2). This represents the first, independent replication of previously observed longitudinal increases in SN FW in IPD, ${ }^{10,11}$ though in the aSN segment and not the pSN. These data, in total, support SN FW as a promising disease-progression biomarker in IPD.

As noted, previous studies have shown conflicting results regarding cross-sectional, conventional DTI assessments of the SN in subjects with IPD compared with HCs. ${ }^{5,26}$ These inconsistencies may, in part, be due to FW contamination of the conventional DTI measures ${ }^{7-9,27}$ or to discrepancies in SN ROI delineation. ${ }^{5}$ Both cross-sectional and longitudinal studies, including ours, have shown SN FW to distinguish those with IPD from HCs better and to be more sensitive to SN changes over time than conventional DTI or FW-corrected DTI SN assessments..$^{8-11}$ Another recent longitudinal DTI study by Loane et $\mathrm{al}^{28}$ did not find any cross-sectional differences in SN fractional anisotropy or mean diffusivity at baseline between subjects with IPD and HCs but did find significant longitudinal changes in both assessments in IPD over about 1.5 years of follow-up. However, the Loane et al study found that SN fractional anisotropy values in HCs at baseline fell between SN fractional anisotropy values in subjects with IPD at baseline and those with IPD 1.5 years later. Such a finding would not be consistent with a valid IPD disease state biomarker. On the other hand, when using SN FW assessments, which were not used by Loane et al, our study and previous studies have shown baseline intergroup differences consistent with a disease state biomarker and showed SN FW to significantly increase longitudinally. ${ }^{10,11}$

It also appears that SN ROI delineation may affect DTI study results in IPD. For example, Langley et $\mathrm{al}^{5}$ showed robust crosssectional differences in SN fractional anisotropy using neu- 
Table 1: Subject demographic and clinical information ${ }^{a}$

\begin{tabular}{|c|c|c|c|c|c|c|}
\hline & \multicolumn{2}{|c|}{$\mathrm{HC}(n=19)$} & \multicolumn{2}{|c|}{ IPD $(n=19)$} & \multicolumn{2}{|c|}{ Between Groups } \\
\hline & BL & $F / U$ & BL & $F / U$ & $P$ Value within $B L$ & $P$ Value within $\mathrm{F} / \mathrm{U}$ \\
\hline Age (yr) & $56.5(10.1)$ & $59.6(10.3)$ & $59.8(8.4)$ & $63.2(8.4)$ & .276 & .251 \\
\hline Male sex (No.) (\%) & $5(26)$ & - & $12(63)$ & - & .022 & - \\
\hline Disease duration (yr) & - & - & $7.1(5.1)$ & $10.4(5.3)$ & - & - \\
\hline Time to F/U (mo) & - & $36.2(5.4)$ & - & $43.8(7.8)$ & - & .001 \\
\hline MMSE score & $29.4(0.9)$ & $29.5(0.9)$ & $29.4(1.0)$ & $27.6(4.8)$ & .586 & .862 \\
\hline MoCA score & $27.3(2.4)$ & $27.9(2.1)$ & $24.4(4.2)$ & $24.3(6.9)$ & .095 & .046 \\
\hline CDR score (mean, median) (IQR) & $0,0(0-0)$ & $0.1,0(0-0)$ & $0.2,0(0-0.5)$ & $0.3,0(0-0.5)$ & .053 & .317 \\
\hline UPDRS-III score & $0.8(1.2)$ & $1.3(2.3)$ & $18.8(7.3)$ & $22.1(9.2)$ & $<.001$ & $<.001$ \\
\hline H\&Y (mean, median) (IQR) & $0,0(0-0)$ & $0,0(0)$ & $1.9,2(1-2.5)$ & $2.4,2.5(2-3)$ & $<.001$ & $<.001$ \\
\hline S\&E score & $100(0)$ & $100(0)$ & $91.3(9.3)$ & $79.5(21.8)$ & .001 & .001 \\
\hline GDS score & $1.9(2.9)$ & $1.2(1.9)$ & $2.3(2.1)$ & $5.2(7.9)$ & .615 & .044 \\
\hline
\end{tabular}

Note:-H\&Y indicates Hoehn and Yahr scale; S\&E, Schwab and England Activities of Daily Living Scale; GDS, Geriatric Depression Scale; IQR, interquartile range; BL, baseline; F/U, follow up; CDR, Clinical Dementia Rating; UPDRS III, Unified Parkinson's Disease Rating Scale, Part III.

a Results are presented as mean (SD), unless otherwise noted.

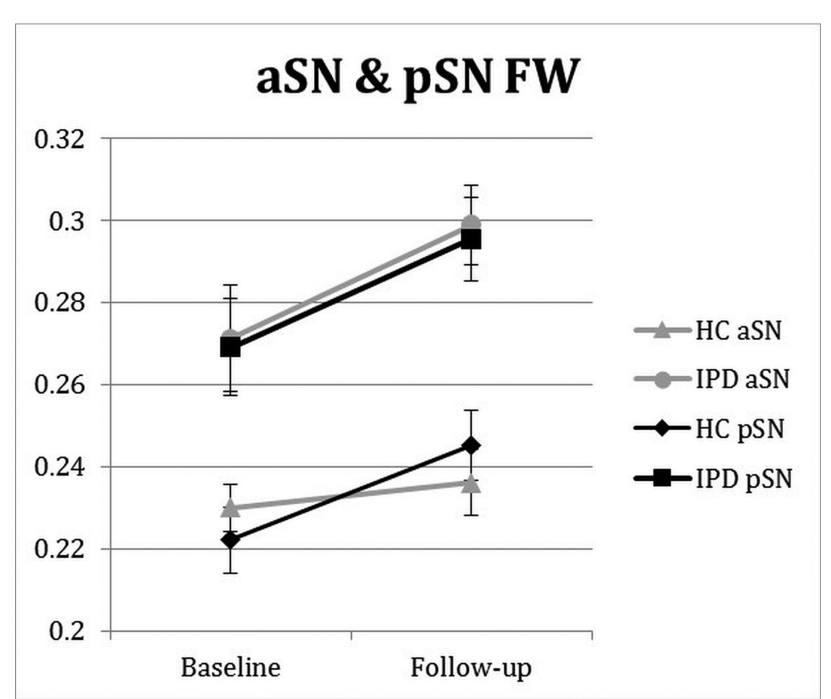

FIG 2. Longitudinal changes in SN FW in patients with later-stage IPD and HCs. aSN and pSN FW group and group $\times$ time interaction intergroup differences were $P<.001$, $=0.021$, and $P=.014,=0.651$, respectively. Follow-up was approximately 3 years from baseline. Error bars are \pm 1 standard error of mean.

romelanin-sensitive $\mathrm{MR}$ imaging to delineate the $\mathrm{SN}$ ROI in a location mostly inferior to the red nucleus but found no significant cross-sectional differences in SN fractional anisotropy when the SN ROI was delineated at the level of the red nucleus in the same IPD and HC cohorts. Therefore, using FW assessments and delineating the SN ROI just inferior to the red nucleus, as was done by Langley et al, by the Vaillancourt group, and by us in the present study (Fig 1), 5,8-11,29 may provide more consistent results in future cross-sectional and longitudinal IPD studies.

The main novel finding in our study was that aSN and not pSN showed significant longitudinal increases in FW. Whether one observes FW longitudinal increases in the pSN and/or aSN may be a reflection of IPD disease duration, with the pSN first showing increased FW followed by the aSN, which would be in keeping with the known spatial profile of SN degeneration over time in IPD. ${ }^{3}$ This possibility is supported by the fact that the 2 IPD cohorts previously studied had mean disease durations at baseline of 0.6 and 3.0 years and showed longitudinal increases in pSN FW, while our IPD cohort had a mean disease duration of 7.1 years at baseline and showed significant longitudinal increases in aSN
FW. ${ }^{10,11}$ Indeed, another IPD cohort with a mean disease duration of 5.2 years showed significant cross-sectional increases in both aSN and pSN FW compared with HCs, similar to findings in our cohort (Fig 2 and Table 2). ${ }^{9}$ Although these data support SN FW to potentially reflect the known posterior-to-anterior pattern of SN degeneration with time in IPD, ${ }^{3}$ further work is needed to better clarify cross-sectional and longitudinal FW differences at both $\mathrm{SN}$ sites based on disease duration in IPD.

Identification of disease-progression biomarkers that are not influenced by IPD symptomatic therapies will be of great value in identifying disease-modifying therapies for IPD. ${ }^{30} \mathrm{SN}$ FW represents a logical biomarker toward this end. Free water is water molecules within a voxel that are not hindered or restricted by the cellular environment and therefore originate from extracellular water. ${ }^{7}$ Free water has been shown to reflect pathologic processes, including tissue atrophy and inflammation, ${ }^{31}$ both of which are known to occur in the SN of IPD. In addition, SN FW is not significantly affected by acute levodopa administration in subjects with IPD. ${ }^{32}$ Thus, SN FW assessments may help to distinguish the disease-modifying effect of a therapy, such as a reduction in SN degeneration and atrophy, from its symptomatic action, which has been difficult to achieve on the basis of clinical outcome assessment alone. ${ }^{30}$ In addition, 3 T MR imaging is widely available and relatively inexpensive to perform compared with radioligand-based imaging modalities. Such features make SN FW assessed by MR imaging an attractive imaging outcome to incorporate into future disease-modifying clinical trials.

It would also be interesting to explore how $\mathrm{SN}$ assessments using newer multicompartmental diffusion imaging modalities, such as neurite orientation dispersion and density imaging (NODDI) or restriction spectrum imaging, compare with SN FW both cross-sectionally and longitudinally in IPD. ${ }^{33,34}$ With different algorithms than we used to assess FW, both NODDI and restriction spectrum imaging also provide estimations of isotropic FW as well as neurite density disentangled from orientation dispersion. $^{33,34}$ Thus, NODDI and restriction spectrum imaging may prove to be more sensitive modalities to assess SN pathology in IPD than the FW assessment technique we have used. In fact, SN NODDI has recently been shown to robustly distinguish those with IPD from HCs in a cross-sectional fashion; however, neither NODDI nor restriction spectrum imaging have been used to assess longitudinal SN changes in IPD, to date. ${ }^{35}$ Also, these techniques require more ad- 
Table 2: SN FW outcomes over 3 years of follow-up in HC and IPD groups ${ }^{\mathrm{a}}$

\begin{tabular}{|c|c|c|c|c|c|c|c|}
\hline & \multicolumn{2}{|c|}{$\mathrm{HC}(n=19)$} & \multicolumn{2}{|c|}{ IPD $(n=19)$} & \multirow[b]{2}{*}{ Group Effect } & \multirow[b]{2}{*}{ Time Effect } & \multirow[b]{2}{*}{ Interaction } \\
\hline & Baseline & Follow-Up & Baseline & Follow-Up & & & \\
\hline aSN FW & $.2301(.0243)$ & $.2361(.0351)$ & $.2713(.0535)$ & $.2989(.0364)$ & $<.001$ & .112 & .021 \\
\hline pSN FW & $.2221(.0349)$ & $.2452(.0367)$ & $.2691(.0513)$ & $.2955(.0441)$ & .014 & .246 & .651 \\
\hline
\end{tabular}

${ }^{a}$ Values are mean (SD). Group, time, and group $\times$ time interaction effect values are $P$ values.

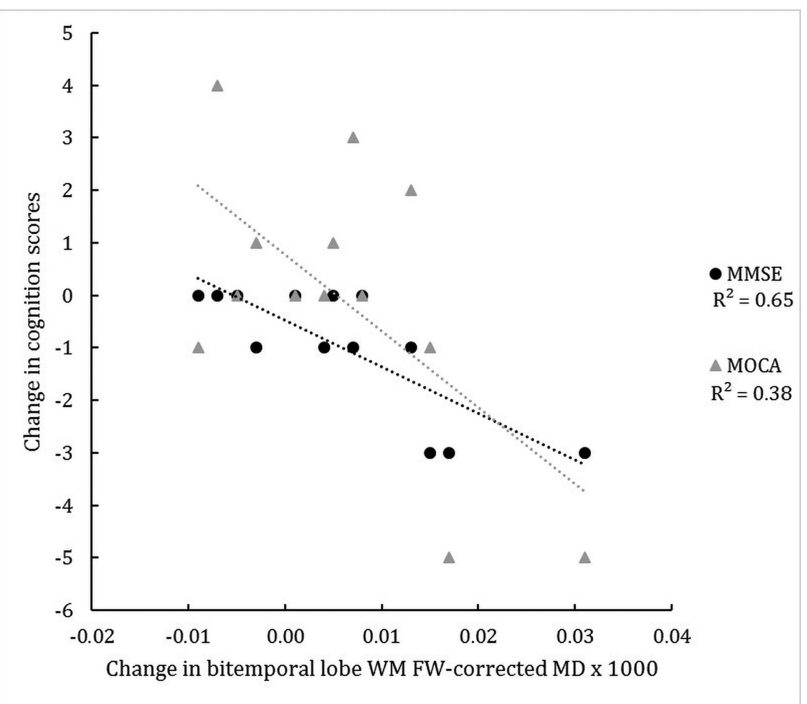

FIG 3. Change in the in bi-temporal lobe white matter FW-corrected mean diffusivity of subjects with IPD and corresponding changes in the MMSE and MoCA scores over 3 years. $R^{2}$ indicates coefficient of determination.

vanced acquisition schemes, whereas the bi-tensor model used in the current study can be applied to any DTI-based protocol.

Besides the significant longitudinal changes in aSN FW, we also found that longitudinal increases in bitemporal white matter FWcorrected mean diffusivity were associated with longitudinal worsening of cognition in IPD as measured by both the MMSE and MoCA (Fig 3). Previous cross-sectional studies have also found significant lobar white matter DTI changes associated with worsened cognition in IPD. ${ }^{19,20,22}$ Meaningful clinical outcomes not responsive to levodopa, such as cognition, will likely be critical to the interpretation of long-term disease-modifying trials because the nonmotor symptom of dementia is typically far more disabling than the IPD motor symptoms. ${ }^{30}$ Because it takes approximately 10 years on average after IPD diagnosis for dementia to occur, ${ }^{36}$ the use of biomarkers that are sensitive to cognitive decline in IPD would help to identify cognitiveprotecting disease-modifying therapies during a shorter time period is more realistic for the duration of a randomized controlled trial. Our findings suggest that longitudinal assessment of temporal lobe white matter FW-corrected mean diffusivity may represent a cognitive biomarker to complement a disease-modifying trial design. However, because this is the first longitudinal study showing this association and our non-SN ROI outcomes were not corrected for multiple comparisons, these results should be considered preliminary until further data are available.

We did not find any significant longitudinal or post hoc crosssectional differences at baseline between the IPD-R and IPD-NM groups for any ROI assessment after correction for the intergroup differences in disease durations. In contrast, a recent study did find significantly reduced pSN FW in subjects with IPD-R with a mean disease duration of 2.3 years compared with subjects with IPD-NM. ${ }^{37}$ The differences in these findings may be due to differences in sample sizes, IPD cohort disease duration, or MR imaging acquisition techniques. A prospective, randomized longitudinal study addressing the effects of rasagiline on pSN FW is currently in progress. ${ }^{38}$

The main weakness of our study was the high rate of subject attrition. This may have been due to the original 57 subjects needing to give consent to a new study after a prolonged, 3-year period and due to the more advanced disease duration of our IPD cohort (7.1 years at baseline). The loss of $>30 \%$ of our original cohort likely diminished our power to detect intergroup differences. The Ofori et $\mathrm{al}^{10}$ and Loane et $\mathrm{al}^{28}$ longitudinal IPD imaging studies used 1- and 1.5-year follow-up periods and IPD cohorts with disease durations at baseline of 3.0 and 3.9 years, respectively, and had no subject attrition, suggesting these to be preferable follow-up periods and IPD disease durations for maximizing subject retention. ${ }^{10,28}$

Another weakness of our study was the use of a 4-mm slice thickness for the DTI-based MR imaging sequence with $0.5-\mathrm{mm}$ gaps between slices and only 25 gradient directions. Most of the previously referenced DTI and FW studies used a $2-\mathrm{mm}$ slice thickness without any gaps and 64 gradient directions. 5,9,10,20,28 The coarser image and angular resolutions from our MR imaging protocol compared with MR imaging protocols of the previous studies likely decreased our sensitivity to detect intergroup differences. Also, the larger MR imaging slice thickness used in our study most likely accounts for the much higher SN FW values, even in HCs, compared with those found in previous studies. ${ }^{9,10}$ Future studies assessing SN FW and/or DTI in IPD would likely benefit from the use of an MR imaging slice thickness close to $2 \mathrm{~mm}$ without gaps between slices and a larger number of gradient directions.

\section{CONCLUSIONS}

This study supports SN FW assessed by MR imaging as a promising disease-progression biomarker in IPD. Now that 2 independent groups have reported significant increases in SN FW over time in 3 different IPD cohorts, further research is justified on longitudinal SN FW changes in IPD and on longitudinal effects of potential diseasemodifying therapies on this neuroimaging outcome.

\section{ACKNOWLEDGMENTS}

We thank David E. Vaillancourt for providing example SN ROIs. We also thank all the research participants.

Disclosures: Thomas Guttuso Jr-RELATED: Grant: Teva Neuroscience, Comments: An investigator-initiated grant supported some of this research*; UNRELATED: Consultancy: Teva Neurosciences, US Worldmeds; Payment for Lectures Including Service on Speakers Bureaus: Teva Neurosciences. David G. Lichter-RELATED: Grant: Teva Neuroscience, Comments: investigator-initiated award to T. Guttuso Jr; UNRELATED: Consultancy: US Worldmeds, Comments: Xadago launch; Payment for Lectures Including Service on Speakers Bureaus: Teva Neuroscience, University of 
Oklahoma Foundation, Comments: Neurology Grand Rounds, University of Oklahoma, Speaker's Bureau, Teva Neuroscience. Ofer Pasternak-RELATED: Grant: National Institutes of Health, Comments: R01MH108574 and P41EB015902*; UNRELATED: Consulting Fee or Honorarium: University of Florida. Robert Zivadinov-RELATED: Grant: Teva Neuroscience *; UNRELATED: Grants/Grants Pending: Sanofi-Genzyme, Novartis, Roche*; Payment for Lectures Including Service on Speakers Bureaus: Sanofi-Genzyme, Novartis, Roche, Celgene. *Money paid to the institution.

\section{REFERENCES}

1. MaSY, Roytta M, RinneJO, et al. Correlation between neuromorphometry in the substantia nigra and clinical features in Parkinson's disease using disector counts. J Neurol Sci 1997;151:83-87 CrossRef Medline

2. Greffard S, Verny M, Bonnet AM, et al. Motor score of the Unified Parkinson Disease Rating Scale as a good predictor of Lewy bodyassociated neuronal loss in the substantia nigra. Arch Neurol 2006; 63:584-88 CrossRef Medline

3. Kordower JH, Olanow CW, Dodiya HB, et al. Disease duration and the integrity of the nigrostriatal system in Parkinson's disease. Brain 2013;136:2419-31 CrossRef Medline

4. Alexander AL, Lee JE, Lazar M, et al. Diffusion tensor imaging of the brain. Neurotherapeutics 2007;4:316-29 CrossRef Medline

5. Langley J, Huddleston DE, Merritt M, et al. Diffusion tensor imaging of the substantia nigra in Parkinson's disease revisited. Hum Brain Mapp 2016;37:2547-56 CrossRef Medline

6. Vaillancourt DE, Spraker MB, Prodoehl J, et al. High-resolution diffusion tensor imaging in the substantia nigra of de novo Parkinson disease. Neurology 2009;72:1378-84 CrossRef Medline

7. Pasternak O, Sochen N, Gur Y, et al. Free water elimination and mapping from diffusion MRI. Magn Reson Med 2009;62:717-30 CrossRef Medline

8. Ofori E, Pasternak O, Planetta PJ, et al. Increased free water in the substantia nigra of Parkinson's disease: a single-site and multi-site study. Neurobiol Aging 2015;36:1097-104 CrossRef Medline

9. Planetta PJ, Ofori E, Pasternak O, et al. Free-water imaging in Parkinson's disease and atypical parkinsonism. Brain 2016;139:495508 CrossRef Medline

10. Ofori E, Pasternak O, Planetta PJ, et al. Longitudinal changes in free-water within the substantia nigra of Parkinson's disease. Brain 2015;138:2322-31 CrossRef Medline

11. Burciu RG, Ofori E, Archer DB, et al. Progression marker of Parkinson's disease: a 4-year multisite imaging study. Brain 2017;140: 2183-92 CrossRef Medline

12. Hely MA, Reid WG, Adena MA, et al. The Sydney multicenter study of Parkinson's disease: the inevitability of dementia at 20 years. Mov Disord 2008;23:837-44 CrossRef Medline

13. Olanow CW, Rascol O, Hauser R, et al; ADAGIO Study Investigators. A double-blind, delayed-start trial of rasagiline in Parkinson's disease. $N$ Engl J Med 2009;361:1268-78 CrossRef Medline

14. Fahn S, Elton RL; UPDRS Development Committee. The Unified Parkinson's Disease Rating Scale. In: Fahn S, Marsden CD, Calne DB, et al, eds. Recent Developments in Parkinson's Disease. Vol 2. Florham Park: Macmillan Healthcare Information; 1987:153-63

15. Gill DJ, Freshman A, Blender JA, et al. The Montreal cognitive assessment as a screening tool for cognitive impairment in Parkinson's disease. Mov Disord 2008;23:1043-46 CrossRef Medline

16. Folstein MF, Folstein SE, McHugh PR. "Mini-mental state": a practical method for grading the cognitive state of patients for the clinician. J Psychiatr Res 1975;12:189-98 CrossRef Medline

17. Gibb WR, Lees AJ. The relevance of the Lewy body to the pathogenesis of idiopathic Parkinson's disease. J Neurol Neurosurg Psychiatry 1988;51:745-52 CrossRef Medline

18. Andersson JL, Sotiropoulos SN. An integrated approach to correc- tion for off-resonance effects and subject movement in diffusion MR imaging. Neuroimage 2016;125:1063-78 CrossRef Medline

19. Deng B, Zhang Y, Wang L, et al. Diffusion tensor imaging reveals white matter changes associated with cognitive status in patients with Parkinson's disease. Am J Alzheimers Dis Other Demen 2013;28: 154-64 CrossRef Medline

20. Duncan GW, Firbank MJ, Yarnall AJ, et al. Gray and white matter imaging: a biomarker for cognitive impairment in early Parkinson's disease? Mov Disord 2016;31:103-10 CrossRef Medline

21. Fletcher E, Raman M, Huebner P, et al. Loss of fornix white matter volume as a predictor of cognitive impairment in cognitively normal elderly individuals. JAMA Neurol 2013;70:1389-95 CrossRef Medline

22. Melzer TR, Watts R, MacAskill MR, et al. White matter microstructure deteriorates across cognitive stages in Parkinson disease. $\mathrm{Neu}$ rology 2013;80:1841-49 CrossRef Medline

23. Planetta PJ, Schulze ET, Geary EK, et al. Thalamic projection fiber integrity in de novo Parkinson disease. AJNR Am J Neuroradiol 2013;34:74-79 CrossRef Medline

24. Zhang Y, Brady M, Smith S. Segmentation of brain MR images through a hidden Markov random field model and the expectation-maximization algorithm. IEEE Trans Med Imaging 2001;20:45-57 CrossRef Medline

25. Fonov V, Evans AC, Botteron K, et al; Brain Development Cooperative Group. Unbiased average age-appropriate atlases for pediatric studies. Neuroimage 2011;54:313-27 CrossRef Medline

26. Schuff $\mathrm{N}, \mathrm{Wu} I \mathrm{IW}$, Buckley $\mathrm{S}$, et al. Diffusion imaging of nigral alterations in early Parkinson's disease with dopaminergic deficits. Mov Disord 2015;30:1885-92 CrossRef Medline

27. Metzler-Baddeley C, O'Sullivan MJ, Bells S, et al. How and how not to correct for CSF-contamination in diffusion MRI. Neuroimage 2012; 59:1394-403 CrossRef Medline

28. Loane C, Politis M, Kefalopoulou Z, et al. Aberrant nigral diffusion in Parkinson's disease: a longitudinal diffusion tensor imaging study. Mov Disord 2016;31:1020-26 CrossRef Medline

29. Vaillancourt DE, Spraker MB, Prodoehl J, et al. Effects of aging on the ventral and dorsal substantia nigra using diffusion tensor imaging. Neurobiol Aging 2012;33:35-42 CrossRef Medline

30. Olanow CW, Kieburtz K. Defining disease-modifying therapies for PD-a road map for moving forward. Mov Disord 2010;25:1774-79 CrossRef Medline

31. Pasternak O, Shenton ME, Westin CF. Estimation of extracellular volume from regularized multi-shell diffusion MRI. Med Image Comput Comput Assist Interv 2012;15(Pt 2):305-12 Medline

32. Chung JW, Burciu RG, Ofori E, et al. Parkinson's disease diffusion MRI is not affected by acute antiparkinsonian medication. Neuroimage Clin 2017;14:417-21 CrossRef Medline

33. Reas ET, Hagler DJ Jr, White NS, et al. Sensitivity of restriction spectrum imaging to memory and neuropathology in Alzheimer's disease. Alzheimers Res Ther 2017;9:55 CrossRef Medline

34. Zhang H, Schneider T, Wheeler-Kingshott CA, et al. NODDI: practical in vivo neurite orientation dispersion and density imaging of the human brain. Neuroimage 2012;61:1000-16 CrossRef Medline

35. Kamagata K, Hatano T, Okuzumi A, et al. Neurite orientation dispersion and density imaging in the substantia nigra in idiopathic Parkinson disease. Eur Radiol 2016;26:2567-77 CrossRef Medline

36. Buter TC, van den Hout A, Matthews FE, et al. Dementia and survival in Parkinson disease: a 12-year population study. Neurology 2008;70:1017-22 CrossRef Medline

37. Burciu RG, Ofori E, Shukla P, et al. Free-water and BOLD imaging changes in Parkinson's disease patients chronically treated with a MAO-B inhibitor. Hum Brain Mapp 2016;37:2894-903 CrossRef Medline

38. Vaillancourt DE. Image Parkinson's Disease Progression Study. https:// clinicaltrials.gov/ct2/show/NCT02789020?term = vaillancourt\&rank=9 Accessed November 24, 2016 